\title{
Cubic with Faster Convergence: An Improved Cubic Fast Convergence Mechanism
}

\author{
Ning Cao \\ Dept. of Computer Science \& Technology \\ East China Normal University \\ Shanghai, China \\ caoning1985@hotmail.com
}

\author{
Wei Zhang \\ Dept. of Computer Science \& Technology \\ East China Normal University \\ Shanghai, China \\ wzhang@cs.ecnu.edu.cn
}

\begin{abstract}
Cubic TCP [1] is a high-speed TCP variant protocol which has been the default TCP implementation in Linux since kernel-2.6.18. In high bandwidth-delay product environment, it can achieve a full utilization of the available bandwidth. However, Cubic TCP has a shortcoming in terms of convergence speed [4]. In this paper, we verify the slow convergence problem of Cubic TCP on testbed. After that, we propose an improved fast convergence mechanism which is called Cubic with Faster Convergence. The feature of this new mechanism is that we use a new bandwidth releasing method instead of simply cut down ssthresh directly. Testbed experiments show that this new mechanism can significantly reduce the convergence time, at the same time, do not affect other performance of Cubic TCP.
\end{abstract}

Keywords-Congestion Control; Cubic TCP; Fast Convergence

\section{INTRODUCTION}

TCP (Transmission Control Protocol) provides a reliable data transmission mechanism. Most of the applications use TCP to transmit data over the Internet. However, it has been reported that standard TCP substantially underutilizes network bandwidth over high-speed and long distance networks [2]. To solve this problem, researchers have been proposing a variety of TCP variant protocols. As the default TCP implementation of Linux since kernel-2.6.18, Cubic TCP is the most widely used TCP variant. It replaces the linear increase function of standard TCP with a cubic increase function, and the increasing of cwnd is RTT independent. Because of these characteristics, the growth of cwnd in Cubic is more aggressive, so it can gain more bandwidth in high bandwidth-delay product environment. But these characteristics also cause the problem of slow convergence i.e. when a new Cubic flow shares the same bottleneck link with an existing Cubic flow, it takes a long time to achieve fair bandwidth allocation. The author of paper [3] verified this shortcoming of Cubic on Dummynet testbed.

Convergence time is an important metric of TCP: with shorter convergence time, new users can get fair network resource faster; also, when a new flow joins into the network, the local stability of this network system is perturbed from its stable state [4], the less convergence time TCP needs means the network system can return toward the locally stable state quicker. So, it not only can improve the fairness of the allocation of network resource, but also can improve the stability of whole network system.
In this paper, an improved Cubic fast convergence mechanism is proposed, which is called Cubic-FC (Cubic with Faster Convergence). This new mechanism does not change the increasing function of Cubic but divides the congestion avoidance into two phases: 1) competition detection phase; and 2) bandwidth releasing phase which uses a new bandwidth releasing method.

The rest of paper is organized as follows: Section II briefly describes Cubic TCP and its fast convergence mechanism. Section III elaborates our new fast convergence mechanism. Section IV gives the detail of the setup of our testbed and experiments result. Finally, conclusions are provided in Section V.

\section{CUBIC TCP}

The most important feature of Cubic TCP is that it uses a cubic window increasing function of the elapsed time from the last windows reduction:

$$
W_{(t)}=C *(t-K)^{3}+W_{\max }
$$

where $\mathrm{C}$ is a Cubic parameter, $\mathrm{t}$ is the elapsed time from the last loss event, and $\mathrm{K}$ is the time period that the above function takes to increase $W_{(t)}$ to $W_{\max }$ when there is no further loss event. $\mathrm{K}$ is calculated by using the following equation:

$$
K=\sqrt[3]{\frac{W_{\text {max }} * \beta}{C}}
$$

where $\beta$ is the window decrease constant.

From the above cubic function, we can learn: when current $c$ wnd is less than $W_{\max }$, the increasing of $c w n d$ is a convex curve; when current $c$ wnd is more than $W_{\text {max }}$, the increasing of cwnd is a concave curve. This divides the growth process of cwnd into "Steady State Behavior" and "Max Probing". The curve of cwnd is shown in Fig. 1.

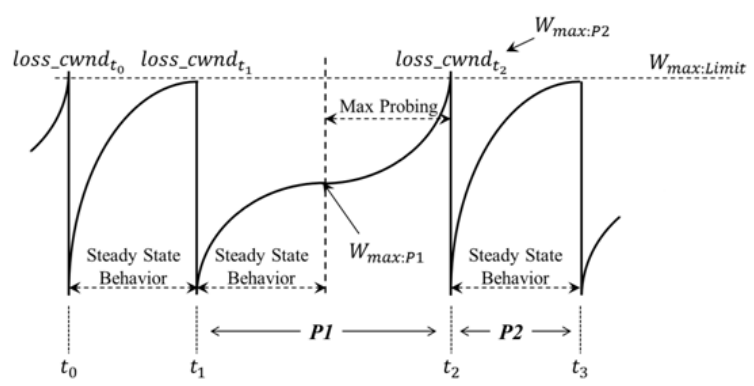

Figure 1. The curve of congestion window of Cubic 


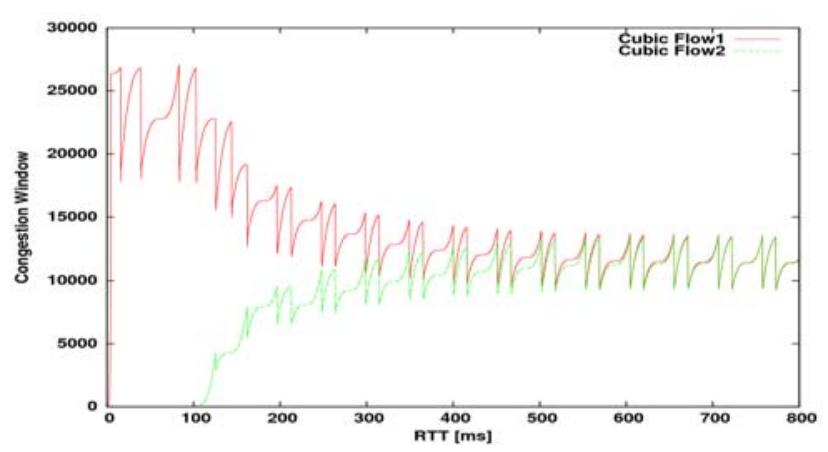

Figure 2. Congestion window size versus time for the original Cubic. Bandwidth of bottleneck link is $800 \mathrm{Mbps}$, RTT is $200 \mathrm{~ms}$, and queue size is $100 \%$ BDP, no web traffic. Flow 1 starts at 0 (s) and ends at $800(\mathrm{~s})$, flow 2 start at $100+x(s)(0<x<1)$ and ends at $800(s)$.

Cubic executes the fast convergence algorithm when congestion is detected. Firstly, it determines whether the cwnd of current loss event (loss_cwnd) is less than the last $W_{\max }:$ if it is, it updates $W_{\max }$ to loss_cwnd $* \frac{2-\beta}{2}$; otherwise, $W_{\max }$ is set to loss_cwnd. After that, Cubic sets ssthresh to loss_cwnd $*(1-\bar{\beta})$, as shown in Fig. 1 .

In a stable network environment, we assume that the maximum $c$ wnd of a TCP flow is $W_{\text {max:Limit }}$ We can learn from the windows growth function of Cubic that when getting close to $W_{\text {max:Limit }}$, cwnd's increasing rate in the "Max Probing" status is greater than it in the "Steady State Behavior" status. In Fig. $1, t_{0}, t_{1}, t_{2}, t_{3}$ are the moments when congestion happens. Before time $t_{1}$, Cubic is in the "Steady State Behavior" status and it is in the "Max Probing" status before time $t_{2}$, the congestion window at time $t_{1}$ is smaller than the congestion window at time $t_{2}$. Also, we can get that $c w n d_{t_{0}}>c w n d_{t_{1}}$ and $c w n d_{t_{2}}>c w n d_{t_{3}}$, which leads that $W_{\text {max }}$ of phase P1: $W_{\text {max:P1 }}=c w n d_{t_{1}} * \frac{2-\beta}{2}$, and Wmax of phase P2: $W_{\text {max }: P 2}=c w n d_{2}$. This kind of effect is mutual which leads to the periodical execution of these two different kinds of growth.

We took an experiment on our testbed to test the convergence time of Cubic. Fig. 2 shows the curves of cwnd of two Cubic flows. As we can see, Cubic needs about 400s to get to the convergence status.

Because flows with larger $c$ wnd's are more aggressive than flows with smaller cwnd's, they can gain more bandwidth than flows with smaller cwnd's within the same time. New flows are thus at a disadvantage and sustained unfairness can occur. "Slow convergence" is considered a general problem in other high-speed TCP variant protocols [5].

\section{CUBIC-FC: CUBIC WITH FASTER CONVERGENCE}

As the description in section II, $W_{\max }$ of phase P2 is greater than phase P1, it leads to the fact that the duration of $\mathrm{P} 2$ is much less than $\mathrm{P} 1$, so $c$ wnd in phase $\mathrm{P} 2$ can arrive to the window limitation faster. Such growth pattern is suitable for maintaining the bandwidth already obtained. As the duration of $\mathrm{P} 1$ is much longer, cwnd needs more time to get to the window limitation, which makes phase P1 suitable for probing competitive flows and releasing bandwidth. We take advantage of this feature of Cubic and divide the congestion avoidance of Cubic into two phases: competition detecting and bandwidth releasing. The cwnd curve of our mechanism is shown in Fig. 3.

\section{A. Competition detection}

In this phase, Cubic-FC detects whether there is (are) competition flow(s) on the bottleneck link, and calculate the decrement of $c w n d(\Delta)$ at the end of this phase, as shown in Fig. 3. If $\Delta$ is bigger than loss_cwnd $* \sigma$, where $\sigma$ is small parameter $(0<\sigma<0.1)$, it means that competition flows may exist on the bottleneck link. In the next bandwidth releasing phase, Cubic will release a certain amount of bandwidth that calculated by this $\Delta$.

As we described before, it takes more time in CP1 phase, flow in this phase would more likely be influenced by shortterm burst, which makes $c$ wnd at $t_{1}$ may not accurately reflect the competition on the bottleneck link. To minimize the possibility of this situation, we use CP2 phase to confirm the decrement of $c w n d$, that is $\Delta=\min \left\{c w n d_{t_{0}}-c w n d_{t_{2}}\right.$, $\left.\Delta_{\max }\right\}$, where $\Delta_{\max }$ is the maximum of the decrement of $c w n d$, and $\Delta_{\max }=$ loss_cwnd $* \beta$

\section{B. Bandwidth releasing}

The convergence speed of TCP depends on two factors [3]: a) the rate at which individual flows release bandwidth when informed of congestion; and b) the rate at which individual flows acquire available bandwidth. The increasing function of Cubic is aggressive enough so that it is not necessary to adopt a more aggressive method. To reduce the convergence time, the only way is to select an appropriate method to release bandwidth. There are two ways to release bandwidth: a) using a bigger decrease parameter when informed of congestion; and b) slowing down the increasing in congestion avoidance. We take advantage of both of these two methods in our mechanism, when detected competition flows:

- $\quad$ Set ssthresh to loss_cwnd $*(1-\beta)-f(\Delta) * \Delta_{\max }$ instead of loss_cwnd $*(1-\beta)$ in original Cubic;

- Set $W_{\max }$ to loss_cwnd $* \frac{2-\beta}{2}-f(\Delta) * \frac{\Delta_{\max }}{2}$ instead

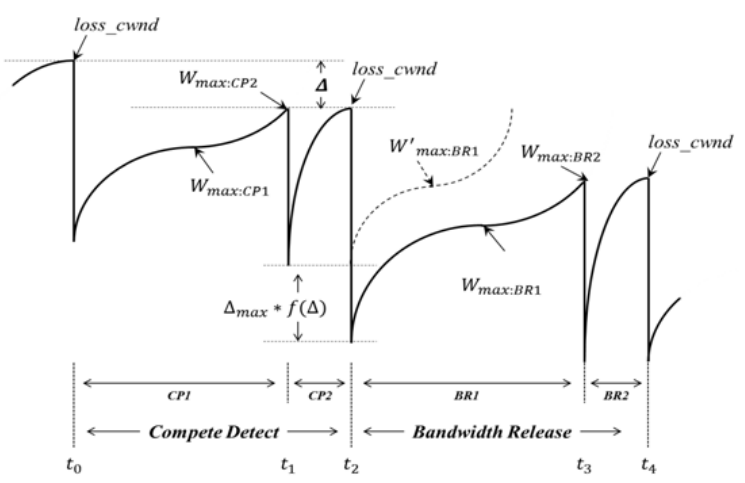

Figure 3. The curve of congestion windows of Cubic-FC 
of loss_cwnd $* \frac{2-\beta}{2}$ in original Cubic;

- Prolong the duration of two congestion event by decrease $t$ to $t *(1-f(\Delta))$, where $t$ is the elapsed time from the last loss event and calculated after receive an ACK.

loss_cwnd is the cwnd of congestion event at the end of last phase, instead of cwnd of last congestion event in original Cubic, as shown in Fig. 3. $\beta$ is the decrease parameter of Cubic as in the original Cubic.

$f(\Delta)$ is a convex function which the range of values is from 0 to $f_{\max }$, where $f_{\max }$ is the maximum of $f(\Delta)$ and $f_{\max }<1$. Also $f(\Delta)$ should meet the following requirements:

- If $\Delta=0$ or $\Delta=\Delta_{\max }$, then $f(\Delta)=0$, and when $\Delta$ gets closer to 0 or $\Delta_{\max }, f(\Delta)$ is closer to 0 ;

- If $\Delta=\Delta_{i}\left(\Delta_{i}\right.$ is a certain value between 0 and $\left.\Delta_{\max }\right)$, $f(\Delta)=f_{\text {max }}$, and $\Delta$ gets closer to $\Delta_{i}, f(\Delta)$ is closer to $f_{\text {max }}$.

The reason $f(\Delta)$ needed these restricts is that:

1) If $\Delta$ is closer to 0 , it means that the reduction of cwnd is more due to normal jitter in the network, we don't need to release bandwidth redundantly;

2) If $\Delta$ is closer to $\Delta_{\max }$, it implies that Cubic has already released an amount of bandwidth of $\Delta$; if $\Delta=\Delta_{\max }$, it may be a multiple packet losses event, and Cubic has already released an amount of bandwidth of $\Delta \max$, it's not necessary to release bandwidth repeatedly.

As shown in Fig. 3, in phase BR1 of bandwidth releasing phase, the dotted line is the window increasing curve of original Cubic, and $\mathrm{W}^{\prime}{ }_{\max } \mathrm{BR} 1$ is the $W_{\max }$ of original Cubic in this phase.

After releasing bandwidth in BR1 of bandwidth releasing phase, Cubic needs to maintain the bandwidth and restrain the growth of the competition flows. Otherwise, as the window growth of Cubic is quite aggressive, the new Cubic flow may in turn exceed the old flow, leads to a new unfairness. To achieve this, we simply set $W_{\max }$ of BR2 phase to the cwnd of congestion event at the end of BR1 phase and set $f(\Delta)$ to 0 that makes Cubic increasing $c w n d$ as aggressive as original algorithm.

\section{EXPERIMENT RESULT}

In this section, we present the detail of our testbed and the experiment. For each scenario, we took at least 10 times and calculated average value.

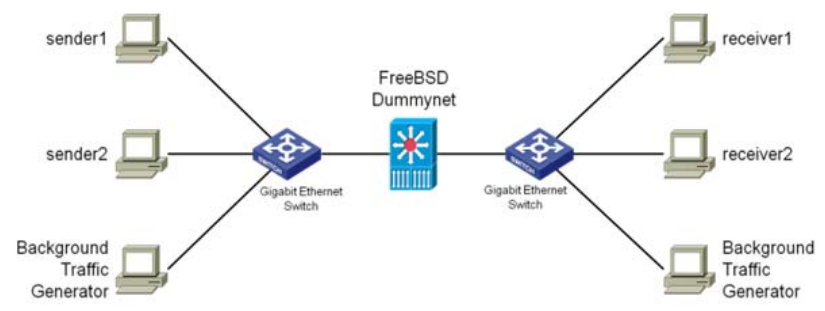

Figure 4. Topology of dummynet testbed
TABLE I. System configuration of our testbed

\begin{tabular}{|l|l|}
\hline CUP & Intel Core i5-2310 2.9GHz \\
\hline Memory & 4Gbyte \\
\hline NIC & $\begin{array}{l}\text { Intel Pro 1000PT DUAL PORT SERVER } \\
\text { ADAPTER }\end{array}$ \\
\hline NIC Driver & e1000 7.1.7 \\
\hline Txqueuelen & 1000 \\
\hline Max backlog & 2500 \\
\hline TX \& RX Descriptors & 4096 \\
\hline
\end{tabular}

\section{A. Testbed setup}

The experiment testbed we are using is a network with a dumbbell topology, see Fig. 4. There are three machines running Linux 2.6.32 with identical hardware and software configurations at each edge of the network. Iperf [6] is used to generate TCP traffic in the senders and receivers. The rest two machines are used to generate background traffic by Surge [7] and Iperf. The amount of background traffic is about $10 \%$ of the bandwidth bottleneck link in both forward and backward directions. The router is running the FreeBSD Dummynet software. In our experiments, the bandwidth of the bottleneck router is set to $800 \mathrm{Mbps}$, and RTT is varied from $50 \mathrm{~ms}$ to $300 \mathrm{~ms}$. The bottleneck buffer size is set to $100 \%$ BDP. We use the drop-tail router at the bottleneck.

The detail of system configuration is shown in Table I.

The function $f(\Delta)$ is an important factor for Cubic-FC. In our experiments, we simply chose a linear function as follow:

$$
f(\Delta)=\left\{\begin{array}{c}
f_{\max } * \frac{\Delta}{\Delta_{i}}, \text { if } \Delta \leq \Delta_{i} \\
f_{\max } * \frac{\Delta_{\max }-\Delta}{\Delta_{i}}, \text { if } \Delta>\Delta_{i}
\end{array}\right.
$$

where $\Delta_{i}=\frac{\Delta_{\max }}{2}$ and $f_{\max }=0.6$. And also, in competition detection phase, the small parameter $\sigma$ which is used to determine whether there are competition flows is set to 0.05 .

\section{B. Convergence Time}

First of all, we evaluated the convergence time of Cubic$\mathrm{FC}$ in the same scene as the experiment in section II. Fig. 5 shows the congestion window size for two Cubic-FC flows.

Compare to original Cubic which needs about 400s to

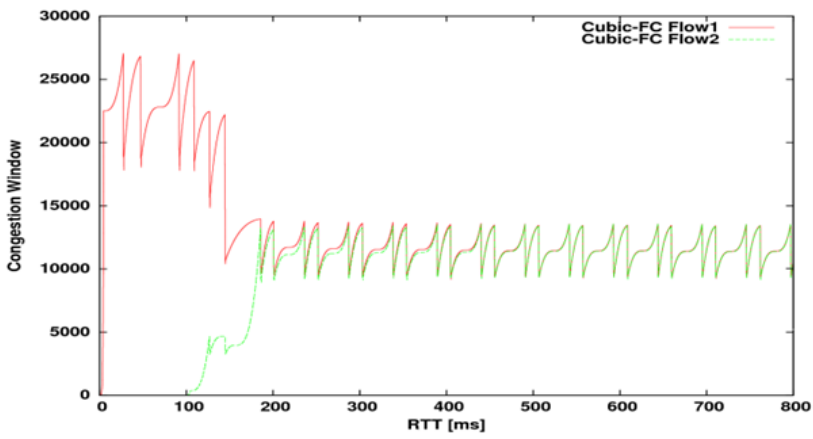

Figure 5. Congestion window size versus time for the Cubic-FC. Bandwidth of bottleneck link is $800 \mathrm{Mbps}$, RTT is $200 \mathrm{~ms}$, and queue size is $100 \%$ BDP, no web traffic. 


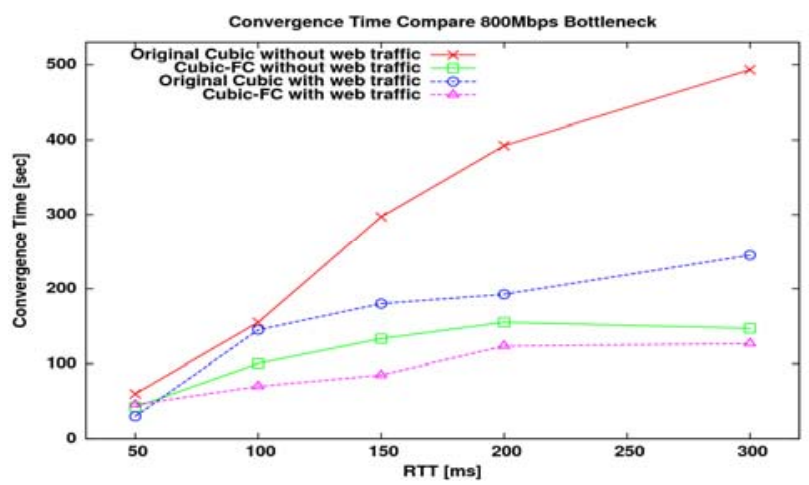

Figure 6. Convergence time compare between original Cubic and CubicFC. Bandwidth is $800 \mathrm{Mbps}$, the running time is $600 \mathrm{~s}$, and RTT is varied from $50 \mathrm{~ms}$ to $300 \mathrm{~ms} .100 \% \mathrm{BDP}$

achieve to the convergence state as shown in Fig. 2, CubicFC flows just need less than 200s.

We also took a series of experiments to confirm the improvement of our mechanism. In each experiment, flow 1 starts at $0(\mathrm{~s})$ and ends at $600(\mathrm{~s})$, flow 2 start at $100+\mathrm{x}(\mathrm{s})$ $(0<x<1)$ and ends at $600(s)$. Fig. 6 shows the convergence times with and without background traffic. We can see Cubic-FC needs shorter time to achieve convergence than original Cubic, and with the RTT gets bigger, Cubic-FC can achieve more improvement. In Fig. 6 we can also see that even background traffic breaks the global synchronization, Cubic-FC can converge faster than original Cubic.

\section{Throughput}

Convergence time can affect the throughput of TCP flows, as the shorter convergence time they need, two TCP flows can achieve fairer throughput.

Fig. 7 shows us the difference of the average throughput of two flows, i.e. average throughput of Flow 1 - average throughput of Flow 2. We can see that, whether with or without background traffic, the two flows of Cubic-FC always can achieve fairer throughput than original Cubic.

To test the impact to the total throughput in our fast convergence mechanism, we measured the total throughput of original Cubic and Cubic-FC in different scenes.

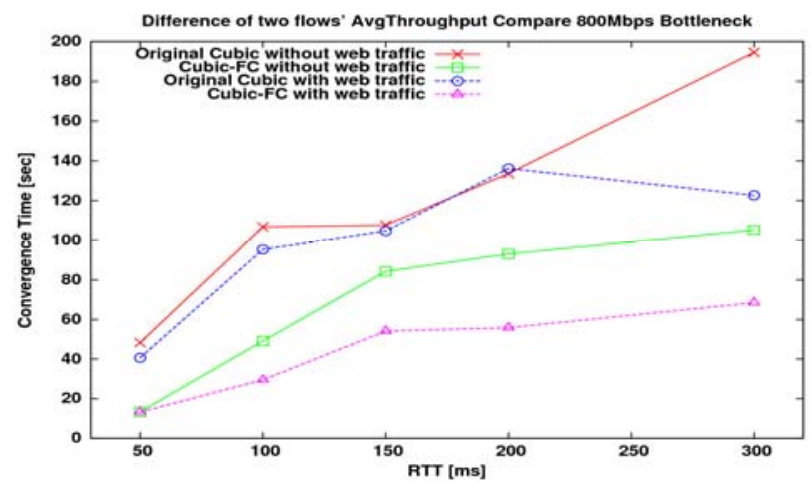

Figure 7. Average throughput of two flows compare between original Cubic and Cubic-FC. Bandwidth is $800 \mathrm{Mbps}$, the running time is $600 \mathrm{~s}$, and RTT is varied from $50 \mathrm{~ms}$ to $300 \mathrm{~ms} .100 \% \mathrm{BDP}$
TABLE II. Total Throughput Compare

\begin{tabular}{|c|c|c|c|c|}
\hline \multirow{2}{*}{ RTT (ms) } & \multicolumn{4}{|c|}{ Total Throughput (Mbps) } \\
\cline { 2 - 5 } & Without background traffic & \multicolumn{2}{c|}{ With background traffic } \\
\cline { 2 - 5 } & Cubic & Cubic-FC & Cubic & Cubic-FC \\
\hline 50 & 772.173 & 772.058 & 685.203 & 680.896 \\
\hline 100 & 772.259 & 772.062 & 685.68 & 681.452 \\
\hline 150 & 772.590 & 772.181 & 686.053 & 683.176 \\
\hline 200 & 772.334 & 772.135 & 684.754 & 681.869 \\
\hline 300 & 772.814 & 772.057 & 683.844 & 682.086 \\
\hline
\end{tabular}

Table II shows the compare of total throughput of two mechanisms. We can see our mechanism does not make any serious impact of the total throughput: in the environment without background traffic, the maximum decrement of total throughput is only $0.098 \%$, and the average value is only $0.014 \%$; in the environment with background traffic, these two values are $0.63 \%$ and $0.46 \%$, respectively. As the duration of two congestion event is longer and congestion window grows slower in bandwidth release phase of CubicFC, the total throughput of Cubic-FC is little smaller than original Cubic, but it is worthy that the slight decline of total throughput in exchange the upgrade of convergence speed.

\section{CONCLUSIONS}

This paper has proposed a new convergence mechanism of Cubic TCP, named Cubic with Faster Convergence (Cubic-FC for short). We divided the congestion avoidance into two phases: competition detection phase and bandwidth releasing phase. In competition detection phase, Cubic-FC detects the competition flows, and in bandwidth releasing phase, we use a new bandwidth releasing method to release a certain amount of bandwidth according to the decrement of cwnd in competition detection phase.

We used testbed rather than simulation, which is closer to realistic network environment. Testbed experiments show that Cubic-FC can converge faster than original Cubic in the environment with and without background traffic, at the same time, it do not seriously impact to the other performance of Cubic.

\section{REFERENCES}

[1] S. Ha, I. Rhee, and L. Xu, "CUBIC: A new TCP-friendly high-speed TCP variant," ACM SIGOPS Operating Systems Review, vol. 42, no. 5, pp. 64-74, 2008.

[2] S.Floyd, "RFC 3649: HighSpeed TCP for Large Congestion Windows", RFC 3649, Experimental, December 2003.

[3] D. Leith, R. Shorten, and G. McCullagh. Experimental evaluation of cubic-TCP. In Proc. Protocols for Fast Long Distance Networks 2007, 2007.

[4] D. Papadimitriou, M. Welzl, M. Scharf and B. Briscoe, "RFC 6077: Open Research Issues in Internet Congestion Control", RFC 6077, INFORMATIONAL, February 2011.

[5] Yee-Ting Li , Douglas Leith and Robert N. Shorten, "Experimental evaluation of TCP protocols for high-speed networks", IEEE/ACM Transactions on Networking (TON), v.15 n.5, p.1109-1122, October 2007.

[6] Iperf. http://sourceforge.net/projects/iperf .

[7] Barford, P., and Crovella, M. Generating representative web workloads for network and server performance evaluation. In Measurement and Modeling of Computer Systems (1998), pp. 151160. 